\title{
A twins heritability study on alpha hemoglobin stabilizing protein (AHSP) expression variability
}

\begin{abstract}
Cytotoxic precipitation of free $\alpha$-globin monomers and its production of reactive oxygen species cause red cell membrane damage that leads to anemia and eventually ineffective erythropoiesis in $\beta$-thalassemia. Alpha hemoglobin stabilizing protein (AHSP) was found to bind only to free $\alpha$-globin monomers creating a stable and inert complex which remains soluble in the cytoplasm thus preventing harmful precipitations. Alpha hemoglobin stabilizing protein was shown to bind nascent $\alpha$-globin monomers with transient strength before transferring $\alpha$-globin to $\beta$-globin to form hemoglobin tetramer. A classical twin study would be beneficial to investigate the role of genetics and environment in the variation of alpha hemoglobin stabilizing protein expression as this knowledge will enable us to determine further investigations with regards to therapeutic interventions if alpha hemoglobin stabilizing protein is to be a therapeutic agent for $\beta$-thalassemia. This study investigates the heritability influence of alpha hemoglobin stabilizing protein expression and factors that may contribute to this. Results indicated that a major proportion of alpha hemoglobin stabilizing protein expression was influenced by genetic heritability $(46 \%)$ with cis-acting factors accounting for $19 \%$ and trans-acting factors at $27 \%$.
\end{abstract}

Keyword: Twins study; Alpha hemoglobin stabilizing protein; AHSP; Phenotypic variance 